\section{IDDF2020-ABS-0168 PREVALENCE OF ELEVATED LIVER ENZYME IN COVID-19 AND ITS ASSOCIATION WITH DISEASE OUTCOME}

Ignatius Bima Prasetya*, Nata Pratama Hardjo Lugito, Andree Kurniawan. Department of Internal Medicine, Faculty of Medicine Pelita Harapan University, Indonesia

\subsection{6/gutjnl-2020-IDDF.14}

Background Coronavirus Disease 19 (Covid-19) had previously been thought to affect mainly respiratory organs. Recent studies had shown that this disease might impact another organ as well, including the liver. Coronavirus has recently been found to be able to bind angiotensin-converting enzyme 2 (ACE2) on cholangiocytes, Leading to cell dysfunction and inflammatory response leading to liver injury. Previous studies had shown that abnormal liver function can be detected in 14\% to $53 \%$ of Covid-19 cases and was related to increased risk of mortality. We aim to evaluate liver enzyme abnormality in Covid-19 patients and its association with disease outcome.

Methods We conducted a retrospective analysis of all confirmed Covid-19 cases from hospitals of Siloam Hospital Group in Indonesia from 6 March until 15 July 2020. Data with unavailable liver enzyme were excluded.

Results We collected data from 266 patients with positive rtPCR for Covid-19. A total of 81 patients (30.5\%) had elevated liver enzyme on admission, with abnormal liver enzyme was defined as serum Alanine Aminotransferase (ALT) value $>35 \mathrm{IU} / \mathrm{L}$. Median ALT was $19 \mathrm{IU} / \mathrm{L}$ in the normal ALT group and $52 \mathrm{IU} / \mathrm{L}$ in the elevated ALT group. Patients in both groups had similar characteristics in age (median 44 vs 47 year-old) and sex distribution (male percentage 69.1\% vs $46.5 \%$ ). The total mortality rate from all patient was $8.3 \%$. Risk of mortality was higher in patients with elevated ALT on admission compared to those with normal ALT $(11.1 \%$ vs 7.0\%, OR: 1.65, 95\% CI: 0.67-4.04, p: 0.266) but this increase is not statistically significant. Patients with elevated ALT also had a statistically significant higher risk of Intensive Care Unit admission $(21.0 \%$ vs $8.6 \%$, OR: 2.81, 95\% CI: $1.34-5.88, \mathrm{p}<0.01)$. The average length of stay was similar between both groups (median 11 days vs 11 days, p: 0.612).
Conclusions Elevated liver enzyme on admission could be found in a significant proportion of Covid-19 patients and was associated with non-statistically significant increased risk of mortality.

\begin{tabular}{ll}
\hline IDDF2020-ABS-0200 & IMPACT OF RISING PREVALENCE OF \\
& METABOLIC COMORBIDITIES ON \\
& ACCURACY OF HEPATOCELLULAR \\
& CARCINOMA RISK SCORE IN CHRONIC \\
& HEPATITIS B PATIENTS RECEIVING \\
& ANTIVIRAL THERAPY: A TERRITORY-WIDE \\
& COHORT OF 31,953 TREATED PATIENTS
\end{tabular}

Terry Cheuk-Fung Yip*, Vincent Wai-Sun Wong, Henry Lik-Yuen Chan, Yee-Kit Tse, Grace Lai-Hung Wong. Department of Medicine and Therapeutics, Medical Data Analytic Centre (MDAC), Institute of Digestive Disease, The Chinese University of Hong Kong, Hong Kong

\subsection{6/gutjnl-2020-IDDF.15}

Background Patients with chronic hepatitis $\mathrm{B}(\mathrm{CHB})$ are getting older with the rising prevalence of metabolic comorbidities. We examined its impact on the performance of PAGE-B score among treated $\mathrm{CHB}$ patients.

Methods Adult $\mathrm{CHB}$ patients who had received entecavir or tenofovir for at least 6 months in 2005-2018 were identified in Hong Kong. Diabetes mellitus (DM), hypertension and dyslipidaemia were identified based on diagnosis codes, laboratory measurements, and medication uses. Performance of PAGE-B score on 5-year HCC prediction was assessed by area under the time-dependent receiver operating characteristic curve (AUROC), and the score's cut-off values were evaluated by survival analysis.

Results Of 31,953 identified CHB patients, there was a trend of rising prevalence of metabolic comorbidities over the three periods (2005-2009, 2010-2014, and 2015-June 2018): hypertension $34.2 \%, 40.3 \%$, and $43.0 \%$; DM 15.2\%, 20.0\%, and 21.6\%; and dyslipidaemia 46.6\%, 55.7\%, and 62.5\%, respectively (all $\mathrm{P}<0.001)$. The AUROC $(95 \% \mathrm{CI})$ of PAGE-B score to predict HCC at 5 years was comparable across the

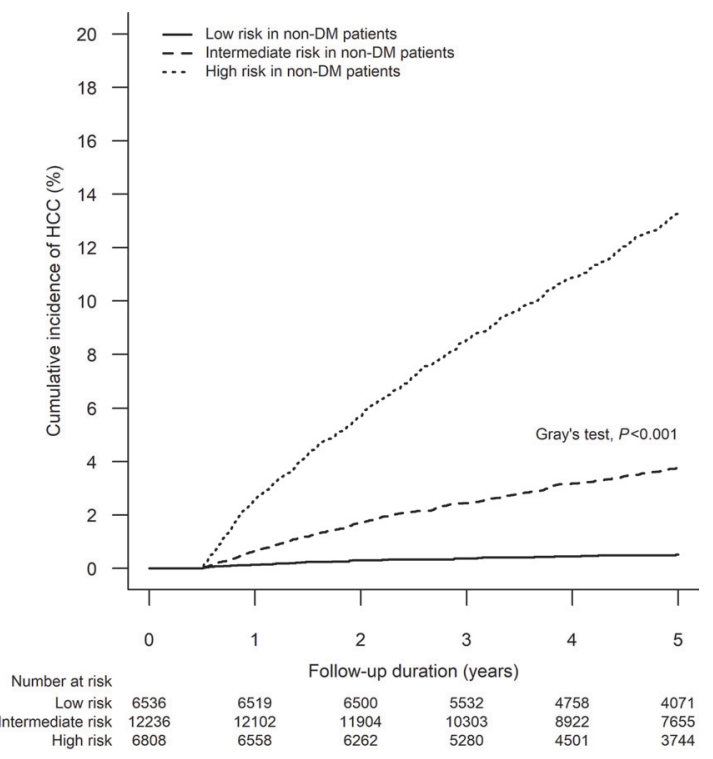

Abstract IDDF2020-ABS-0200 Figure 1 Cumulative incidence function of hepatocellular carcinoma (HCC) in chronic hepatitis B patients on antiviral therapy with and without diabetes mellitus (DM) in different risk groups of PAGE-B score 
three periods: $0.78(0.75-0.80), 0.77(0.76-0.79)$, and 0.75 (0.72-0.78), respectively $(P=0.334)$. Overall, 6,373 (19.9\%) patients had DM. At a median (interquartile range) follow-up of $5.0(3.5-5.0)$ years, $543(8.5 \%)$ and 1270 (5.0\%) patients with and without DM developed HCC. The AUROC $(95 \%$ $\mathrm{CI})$ of PAGE-B score to predict HCC at 5 years was lower in DM patients $(0.68[0.66-0.70])$ than in non-DM patients (0.79 [0.78-0.80]) $(P<0.001) .651(10.2 \%)$ DM patients were classified as low risk; their 5-year HCC cumulative incidence (95\% CI) was $2.1 \%(1.1 \%-3.6 \%)$, which is higher than the threshold of cost-effective HCC surveillance suggested by international clinical guideline, i.e. $0.2 \%$ annually. 6,536 (25.6\%) non-DM patients were classified as low risk; their 5year HCC cumulative incidence $(95 \% \mathrm{CI})$ was $0.5 \%(0.4 \%$ $0.7 \%$ ) (figure 1).

Conclusions PAGE-B score is accurate across the years to predict HCC, yet has a lower performance in DM patients. HCC risk persists and HCC surveillance is still cost-effective in DM patients classified as low risk by PAGE-B score.

\section{IDDF2020-ABS-0206 PREDICTORS OF RESPONSE TO THERAPY WITH TERLIPRESSIN AND ALBUMIN IN PATIENTS WITH CIRRHOSIS AND HEPATORENAL SYNDROME - ACUTE KIDNEY INJURY (HRS-AKI) ACCORDING TO NEW INTERNATIONAL CLUB OF ASCITES (ICA) CRITERIA}

Jatin Agrawal ${ }^{*}$, Ashish Kumar, Anil Arora, Praveen Sharma, Vikas Singla, Naresh Bansal, Shrihari Anikhindi. Institute of Liver, Gastroenterology and Pancreaticobiliary Sciences, Sir Ganga Ram Hospital, India

\subsection{6/gutjn--2020-IDDF.16}

Background HRS-AKI or HRS -1 carries high short-term mortality in patients with advanced cirrhosis. Recently ICA has proposed new definition criteria for HRS, and at present, we lack literature on the response rate and predictors of response of terlipressin according to this new definition. So, we aimed to evaluate the response rate of terlipressin and factors affecting the response rate according to this new HRS-AKI definition

Methods We performed a prospective study on 114 cirrhotic patients with HRS- AKI diagnosed according to ICA definition 2015 from August 2018 to April 2020 using terlipressin and albumin. Baseline clinical and biochemical details were noted. Response was defined as improvement in serum creatinine within $0.3 \mathrm{mg} / \mathrm{dl}$ of baseline (if baseline s.cr $<1.5 \mathrm{mg} / \mathrm{dl}$ ) or $<1.5 \mathrm{mg} / \mathrm{dl}$ (if baseline s.cr $>1.5 \mathrm{mg} / \mathrm{dl}$ ). Further responder and non-responder were followed up to 90 days or death. Univariate and multivariate logistic regression was applied to detect predictors of response. Survival analysis was used to determine 90 days of survival.

Results Among 114 HRS-AKI patients, the median age was 52.5 years, and $83.3 \%$ were male. Response to terlipressin was seen in $70(61.4 \%)$ patients. On subgroup analysis, the response rate in acute decompensation (AD) was seen in 37 $(78.7 \%)$ patients and in acute on chronic failure (ACLF) was seen in $32(47.7 \%)$ patients. Independent predictive factors of response to therapy were serum creatinine before start of terlipressin therapy (odds ratio, 0.390; 95\% confidence interval, $0.195-0.780 ; \mathrm{P}=0.008)$ and baseline child-pugh score (CTP) (odds ratio, 0.584; 95\% confidence interval, 0.382-0.894; $\mathrm{P}$ $=0.013)$. Response to therapy was associated with improved
Kaplan-Meier Survival Estimates According to Response

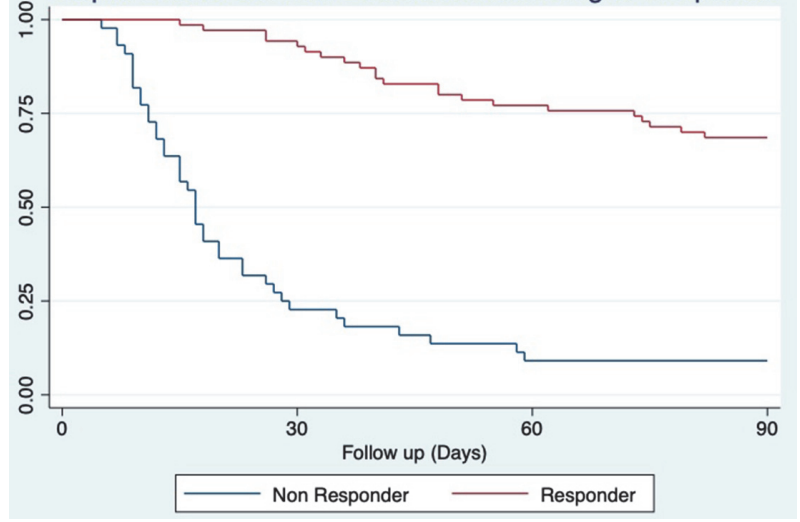

Abstract IDDF2020-ABS-0206 Figure 190 days survival among responder and non- responder groups

90 days of survival compared to patients with non-response (69.56\% vs $10 \%, \mathrm{P}<0.00001)$.

Conclusions Serum creatinine before the start of terlipressin and CTP predicts response to terlipressin in HRS-AKI. The response rate was better with the new definition of HRS. The subset of the population with higher grades of ACLF showed poor response. Therefore early transplantation should be considered in patients of HRS with a low likelihood of response (figure 1).

\section{Basic gastroenterology}

\begin{tabular}{ll}
\hline IDDF2020-ABS-0034 & NETWORK PHARMACOLOGY ANALYSIS TO \\
& UNCOVER THE POTENTIAL MECHANISMS \\
& OF LYCIUM BARBARUM ON COLORECTAL \\
& CANCER
\end{tabular}

Yi Lu*, Jiachen Sun, Minhui Hu, Xianhe Kong, Weijie Zhong. Department of Gastrointestinal Endoscopy, the Sixth Affiliated Hospital, Sun Yat-sen University, China

\subsection{6/gutjnl-2020-IDDF.17}

Background Studies have shown that extracts from lycium barbarum could play a protective role against colorectal cancer (CRC) cells. We used the network pharmacology approach to establish the effects of lycium barbarum on CRC and to predict core targets and their biological functions, pathways, and mechanisms of action.

Methods We obtained the active compounds and their targets in lycium barbarum through Traditional Chinese Medicine System Pharmacology Database (TCMSP), retracted the CRC targets from Malacards, TTD, GeneCards, and DisGeNET, and chosen the overlapped targets as the candidate targets. After protein-protein interaction (PPI) network analysis, 20 with the highest node degree were selected as the core targets, and their enrichment and pathway were analyzed. Furthermore, iGEMDOCK was employed to validate the compound-target association.

Results Eventually, 103 overlapped targets were chosen as the candidate targets. Targets with the top 20 highest node degree were selected as the core targets. Gene Ontology (GO) enrichment analysis indicated that the core targets were significantly enriched in regulation of cell proliferation, extracellular space, cytokine receptor binding, and so on. Kyoto Encyclopedia of 